\title{
Quantitative Sensory Testing to Assessment Objective Changes by Spinal Cord Stimulation In-Patient with Complex Regional Pain Syndrome
}

\author{
Nino Ninidze* and Sabine Sator-Katzenschlager \\ Department of Anesthesiology, General Intensive Care and Pain medicine University of Vienna, Austria
}

*Corresponding author: Nino Ninidze, Department of Anesthesiology, General Intensive Care and Pain medicine University of Vienna, Waehringer Guertel 18-20, A-1090 Vienna, Austria; Tel: ++43 6763619072; Fax: ++43 140400 4165; E-mail: nino.ninidze@meduniwien.ac.at

Received: May 20, 2021; Accepted: May 31, 2021; Published: June 08, 2021

\begin{abstract}
The "complex regional pain syndrome" (CRPS) is characterized by continued pain, debilitating affliction, sensory abnormalities, vaso- and sudomotor disturbances as well as trophic changes. CRPS is often difficult to treat. Spinal cord stimulation (SCS) as a measure to provide adequate pain relief, improve the quality of life and physical function has been employed for that purpose. The sensory profile of the patients with CRPS, including sensory signs: hyperalgesia, allodynia, or hypoesthesia may be linked with the damage and surviving afferent nerve fibres, ectopic impulse generation, peripheral and central sensitization. For comprehensive assessing, the sensory profiles of a patient and results of treatment were verified with quantitative sensory testing (QST) in accordance with the protocol of the German Research Network on Neuropathic Pain (DFNS).
\end{abstract}

Keywords: Complex regional pain syndrome (CRPS), Quantitative sensory testing (QST), Spinal cord stimulation (SCS)

\section{Methods}

Quantitative sensory testing according to the DFNS protocol assesses 13 parameters: cold detection threshold and warm detection threshold (WDT), thermal sensory limen, paradoxical heat sensations (PHS), cold pain and heat pain thresholds, mechanical pain threshold and mechanical pain sensitivity (MPS), dynamical mechanical allodynia (DMA), pressure pain threshold (PPT), wind-up ratio, tactile (mechanical) detection threshold, and vibration detection threshold. Primary outcome parameters, should show comparable difference between active and passive SCS (switched off), after the usage of 'data of the sensitivity with Quantitative Sensory Testing (QST)' of the patient with neuropathic pain by CRPS in at least two distinct mechanisms: sensitization or deafferentation, also baseline demographics, CRPS signs, symptoms, and phenotype (inflammatory, vasomotor, dystonia, edema or neuropathic). Secondary outcome obtaining the full somatosensory phenotype of a patient, including +/-signs (Z-score), for all types of primary afferents, cutaneous and deep pain, peripheral and central sensitization.

\section{Case Report}

A sixty-four-years-old patient was suffering from CRPS I on the left hand. The carpal tunnel syndrome was presented in September 2000 based on distal pain and sensory disturbances in the area of the left hand, with paraesthesia in the first three fingers. In the nerve, examination of the area of the median nerve there was described a distally prolonged latency $(4.7 \mathrm{msec})$. Since August 2002 after 3 Surgeries, there was an intense feeling of cold in the area of the wrist, hypoesthesia in particular in the area of the IV and V finger suffered painful sensations on the radial side in the area of the Metacarpophalangeal joint.

Nerve conduction velocity (NCV): Nerve conduction velocity of the N. Ulnaris of each side was in the normal range. The distal latency of the median nerve of the left hand was prolonged. Previous therapies such as local treatment with Isosorbidedinitraat (Isoket ${ }^{\circ}$ ) therapy, laser therapy, warm Carbonic baths, ultrasound treatment and lymphatic drainage, ultrasound-guided sympathetic blockade, concomitant drug therapy with Celecoxib (Celebrex ${ }^{\circ}$ ) $200 \mathrm{mg}$ Tbl. 1-0-1-0, Amitriptyline (Tryptizol $\left.^{\circ}\right) 75$ mg Tbl. 0-0-0-1, Gabapentin (Neurontin $\left.{ }^{\circ}\right) 300$ mg Kps. 2-2-0-2, Tramundal (Tramal ${ }^{\circ}$ ) ret. $200 \mathrm{mg}$ Tbl. 1-0-0-1, Prednisolone $25 \mathrm{mg}$ Tbl. 1-0-0-0, CT guided radio-frequency Denervation of the ganglion stellatum, had no relevant changes for massive hand back edema, for complete restriction of movement of all fingers, and severe long-term pain. A multidisciplinary pain conference decided to try Spinal Cord Stimulation. One epidural stimulation electrode was implanted on 16.9.03. The tip of the electrode was projected towards the middle of the HWK 3 . The stimulation immediately resulted in a surprisingly strong effect with complete recovery of the edema within 24 hours, freedom from pain under-stimulation, and more complete restoration of finger agility. During follow-up checks, drug therapy reduction became possible.

In 2009 the first battery of the implant system was empty. Under this stopped therapy, the pain came back and also the swelling in the left area of the hand. This was the indication for the exchange of the battery system. A secondary battery change was necessary again in 2018. 


\section{Discussion}

In this case we verified the sensibility with Quantitative Sensory Testing (QST for obtaining the full somatosensory phenotype of this CRPS I patient. The tests were carried out three times. 1. Testing with active stimulation, 2. With stimulation paused for 24 hours and 3. With stimulation reactivated (after 24 hours after activation).

Patient has also shown topical changes on the left hand. With active SCS the patient has no swelling on the left hand (Figure 1), in 24 hours with the SCS switched off the hand was clearly swollen (Figure 2 ) and after 24 hours the reactivation of the SCS, the swelling was again significantly reduced (Figure 3 ).

Significant side differences between the affected and unaffected side were not found in thermal hypoesthesia, mechanical hypoesthesia, and hyperalgesia (Figure 4).

During the test trial of affected side, significant differences were noted when comparing QST results with inactive and active SCS. Patient without SCS (during 24 H SCS break) exhibited the presence of paradoxical heat sensations (PHS), that indicates a disturbance of Ad-cold fiber function (or central pathways encoding for cold sensation), the non-presence of heat hyperalgesia gives evidence for non-peripheral sensitization, whereas the isolated presence of static

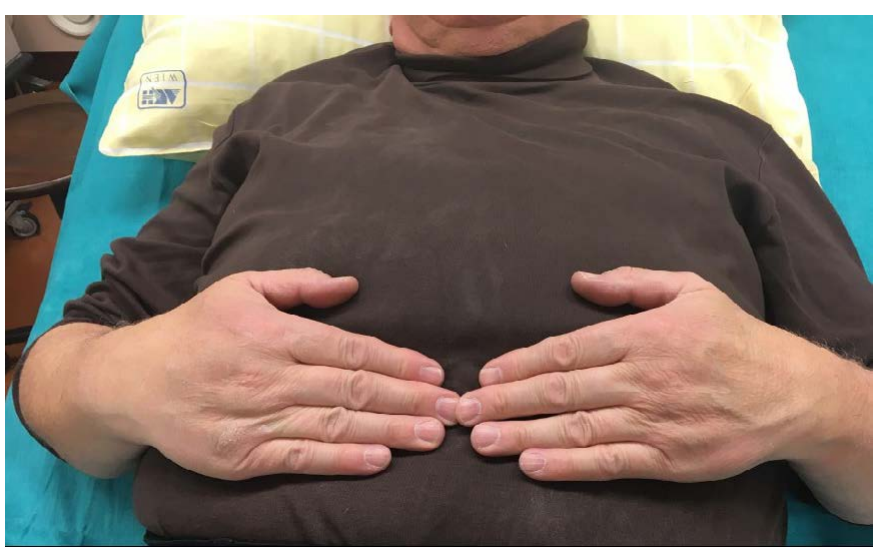

Figure 1: Pat. With CRPS, SCS active.

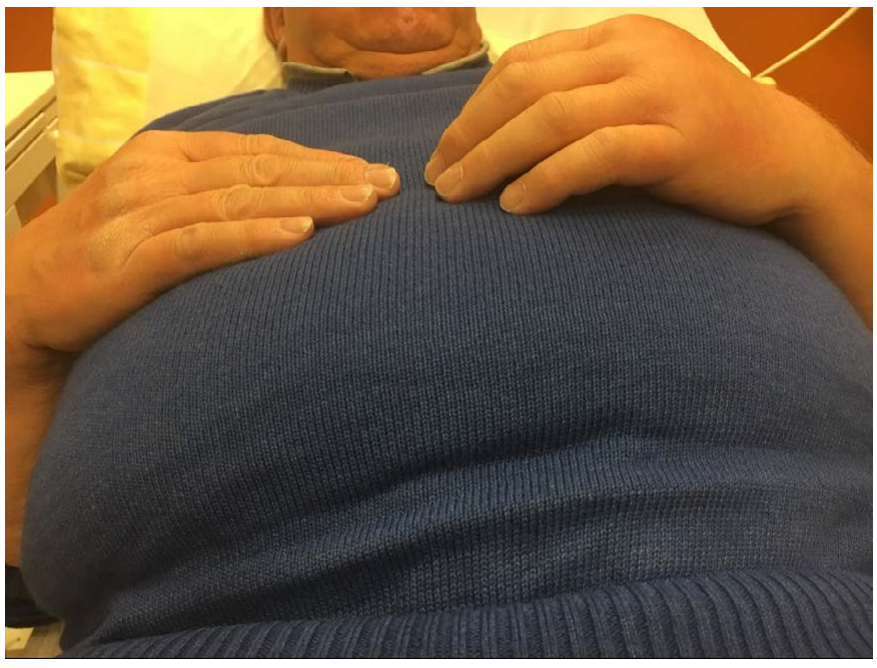

Figure 2: Pat. With CRPS, SCS break, 24H.

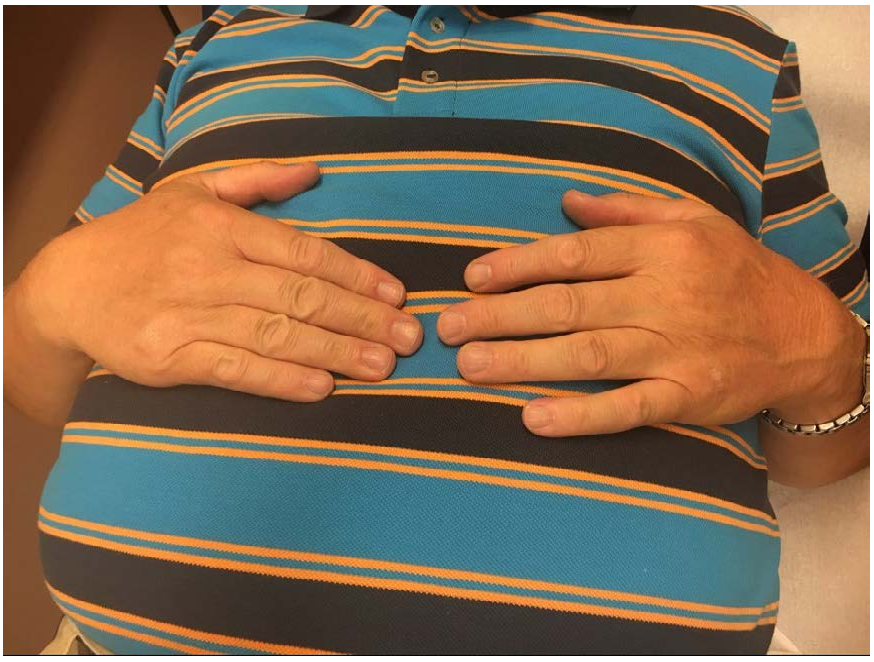

Figure 3: Pat. With CRPS, SCS Active.

mechanical hyperalgesia or dynamic mechanical allodynia (C- and Ad-fiber), hyperalgesia by pressure pain thresholds (PPT) (Figure 5), the Hyperalgesia by Vibration detection threshold (VDT) (Figure 6), the wind-up ratio (WUR) (Figure 7) gives us an indication about central sensitization.

After activating the SCS, the QST data returned closer to the original data. The results are shown in and the figures (Figures 4-7). Quantitative sensory testing allows for standardization of measurement when measuring different Sensation qualities of the skin and peripheral nerve functions. QST is currently used in patients with polyneuropathy of diabetes mellitus or hyperuricemia, after nerve injury and in chronic neuropathic pain syndromes, such as postherpetic neuralgia, trigeminal neuralgia, and post-stroke pain [1]. Comparing these results of this present investigation to data from the literature reveal that there are only few data published concerning sensory measurements during active neurostimulation of the spinal cord. Ruppolt MA and Kress B published data of 7 patients with chronic unilateral radicular neuropathic pain and active Spinal Cord Stimulation [2]. Using measure two consecutive QST measurements for thermal, tactile-static, tactile-dynamic, vibratory and pain sensation of the lower limbs. Measurements were performed when SCS was turned off and once again during SCS and subsequently reduced pain levels.

In contrast, our data Baseline QST demonstrated significantly increased thresholds for warm and cold detection in the pain area. With SCS active, a significant reduction of the cold and warm perception and mechanical detection thresholds was found on the painful side. Youn $\mathrm{Y}$, et al. presented quantitative sensory testing, measured thermal detection and pain thresholds and mechanical detection and pressure pain thresholds, as well as vibratory detection, in 20 SCS patients off stimulation, on traditional stimulation, and HFS in a randomized order [3]. Equal to our results was found nonsignificant differences between OFF, ON, and HFS states were seen in thermal and thermal pain detection. Kemler MA, et al. described in a first randomized controlled trial SCS in CRPS I patients. He demonstrated that with strict selection procedures and successful test stimulation, SCS reduces pain and improves health-related quality of 


\section{UNEFFECTED RIGHT AND LEFT HAND Z-SCORE DATA}

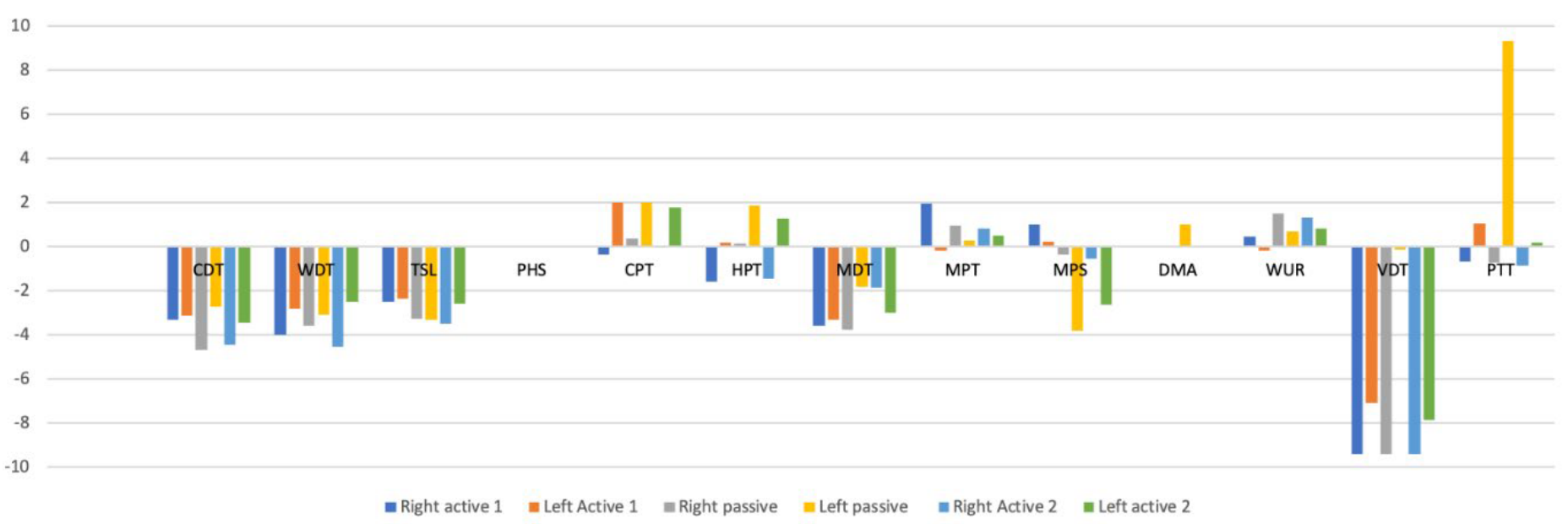

Figure 4: Differences-Score Data between the affected and unaffected side.

AFFECTIVE LEFT HAND. PPT

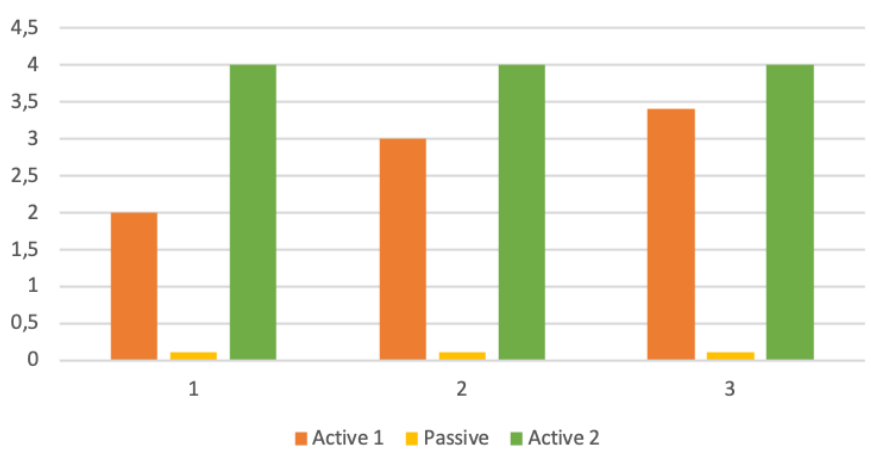

Figure 5: Pressure pain threshold (PPT).

\section{AFFECTIVE LEFT HAND. VDT}

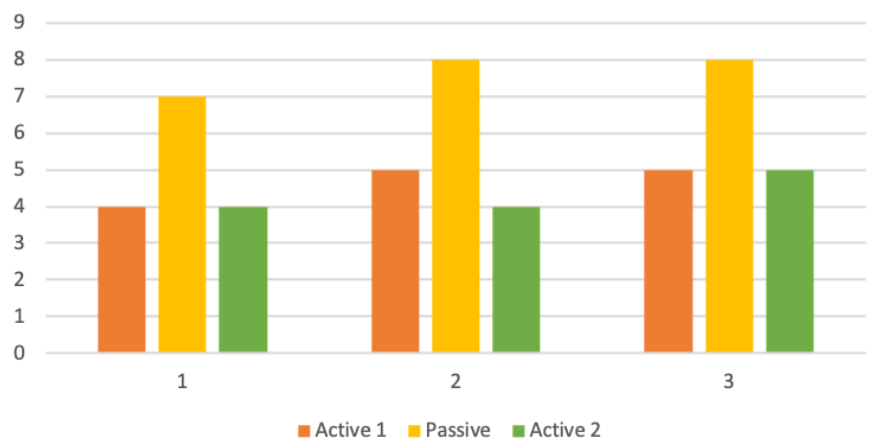

Figure 6: Vibration detection threshold (VDT).

life. "The fact that SCS does not relieve allodynia should be clearly communicated to potential candidates for this treatment"- reported the authors [4]. Our data from our patient showed that SCS does not reduce the intensity of allodynia. In our case, with active stimulation, allodynia by QS Testing was not identified.

\section{Conclusion}

Nociceptor input can trigger a prolonged but reversible increase in the excitability and synaptic efficacy of neurons in central nociceptive
AFFECTED LEFT HAND WUR

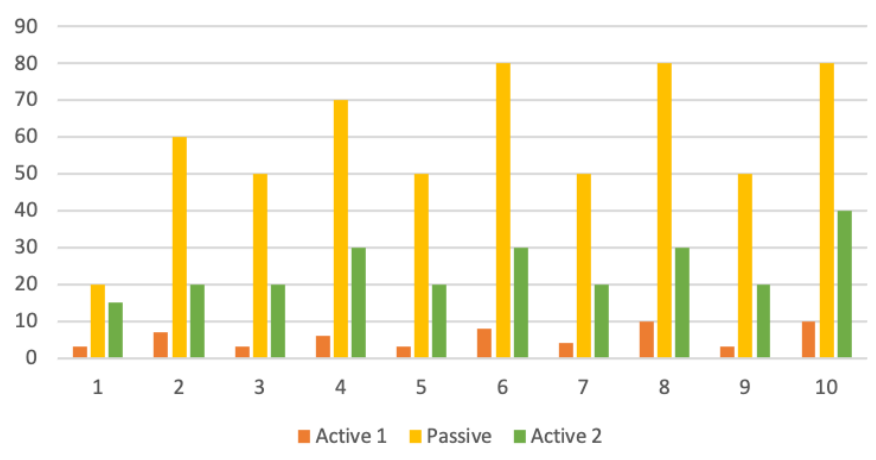

Figure 7: The wind-up ratio (WUR).

pathways, a phenomenon called central sensitization. It manifests as pain hypersensitivity, particularly dynamic tactile allodynia, a secondary pinprick or pressure hyperalgesia, aftersensations, and enhanced temporal summation. Triggering of tingling paresthesia via A- $B$ fibers is a prerequisite for a pain-relieving effect. We had compared the sensitivity of small and long neurons in the skin of the patient with active SCS and stopped SCS. We have seen that patients without stimulation did show a difference in how sensitive they were to things that should hurt, but also ordinary pressure and touch when compared to the same Patient with active SCS. After observing the patient a possible mechanisms of SCS based on the Gate Control Theory could be a peripheral stimulation of A- $B$ fibers that leads a activation of inhibitory interneurons and subsequent inhibition of second-order nociceptive neurons in the dorsal horn and also expand of electrical stimulation of the dorsal column with a production of paresthesia.

We suppose that this may realistically explains the working procedure of SCS, because Allodynia (A- $\beta$ fibers) decreases immediately after starting with the stimulations, but pressure pain (C, A $\delta$ Fiber) and wind-up ratio (C, A $\delta$ Fiber) change later, when the inhibitions response is activated. This phenomenon could also have observed under SCS stimulation and stopped after failing of the battery. 


\section{Authorship Statement}

Dr. Nino Ninidze conducted this case data collection, and data analysis, prepared the manuscript draft. Dr. Nino Ninidze had complete access to the study data and approved the final manuscript.

\section{Conflict of Interest}

Dr. Nino Ninidze and Sabine Sator-Katzenschlager are in no way financially involved or in relationships that might lead to conflict of interests.

\section{References}

1. Baron R, Binder A, Wasner G (2010) Neuropathic pain: diagnosis, pathophysiological mechanisms, and treatment. The Lancet Neurology 9: 807-819. [crossref]

2. Rasche D, Ruppolt MA, Kress B, Unterberg A, Tronnier VM (2006) Quantitative Sensory Testing in Patients with Chronic Unilateral Radicular Neuropathic Pain and Active Spinal Cord Stimulation. Neuromodulation 9: 239-247. [crossref]

3. YounY, Smith H, Morris B, Argoff C, Pilitsis JG (2015) The Effect of High-Frequency Stimulation on Sensory Thresholds in Chronic Pain Patients. Stereotact Funct Neurosurg 93: 355-359. [crossref]

4. Kemler MA, Reulen JP, Barendse GA, van Kleef M, de Vet HC, et al. (2001) Impact of Spinal Cord Stimulation on Sensory Characteristics in Complex Regional Pain Syndrome Type I: A Randomized Trial Anesthesiology 95: 72-80. [crossref] 